\title{
Hypersonic Modes in Nanophononic Semiconductors
}

\author{
S.P. Hepplestone and G. P. Srivastava \\ School of Physics, University of Exeter, Exeter EX4 4QL, United Kingdom
}

(Received 22 April 2008; published 3 September 2008)

\begin{abstract}
Frequency gaps and negative group velocities of hypersonic phonon modes in periodically arranged composite semiconductors are presented. Trends and criteria for phononic gaps are discussed using a variety of atomic-level theoretical approaches. From our calculations, the possibility of achieving semiconductor-based one-dimensional phononic structures is established. We present results of the location and size of gaps, as well as negative group velocities of phonon modes in such structures. In addition to reproducing the results of recent measurements of the locations of the band gaps in the nanosized $\mathrm{Si} / \mathrm{Si}_{0.4} \mathrm{Ge}_{0.6}$ superlattice, we show that such a system is a true one-dimensional hypersonic phononic crystal.
\end{abstract}

DOI: 10.1103/PhysRevLett.101.105502

Phononic crystals (PCs) are the vibrational analogues of photonic crystals and offer the possibility of novel applications for phonon engineering including noise-proof devices and sound filters [1,2]. A PC is created by arranging two or more different materials with contrasting vibrational properties periodically. The acoustic mismatch between the constituent materials can be considered to arise either from difference in interatomic force constants and masses (atomic-scale theory) or in density and elastic modulii (continuum-scale theory). The novel physical properties of such structures arise from the possibility of two important features: creation of phononic band gaps (frequency ranges in which acoustic crystal vibrations cannot propagate) and negative refraction (phonon branches with negative group velocity). These features have been predicted by various theories [3] and have been confirmed by experiments $[2,4]$. Most currently realizable phononic structures rely on solid or fluid composites [2] on the scale of $\mu \mathrm{m}-\mathrm{mm}$, which are poor candidates for electronic and optical applications. Recent technological advances have led to the fabrication of nanophononic materials $[5,6]$, which are potential hypersonic systems. However, from an application point of view, relatively little progress has been made on Si based structures, with most relying on Si-air interfaces [7].

In this Letter, we investigate, by employing at different levels of sophistication and examining available experimental evidence, the criteria to produce nanophononic semiconductor structures. We first apply an easy-tounderstand periodic ball-and-spring model to establish criteria for phononic band gaps. Within the framework of these criteria, we apply an enhanced adiabatic bond-charge model (EBCM) to explore the possibility of semiconducting PCs. Various structures possessing one-dimensional (1D), two-dimensional (2D), and three-dimensional (3D) periodicities are investigated, including the $\mathrm{Si} / \mathrm{SiGe}$ superlattice fabricated by Ezzahri et al. [6]. We produce full phonon dispersion relations and reveal the phononic nature
PACS numbers: 63.22.Np, 43.35.+d, 63.20.-e, 68.35.Iv

of these systems. In particular, we compare our results with the measurements of Ezzahri et al. and reveal important features previously not realized.

The ball-and-spring model provides a sandbox approach for atomic vibrations in a crystal. Previous such models [8] have been restricted to 2D systems with some limitations of either using atomic spacing of $\mu \mathrm{m}$ and masses of grams for systems which are better described using continuum models or only considering small unit cells of up to 4 atoms. For a systematic examination of the criteria and trends, we apply the sandbox approach to systems with various atomic configurations and dimensionalities. For the linear chain model (1D), we consider a composite system consisting of two atom types, $A$ and $B$, with masses $M_{A}$ and $M_{B}$, respectively. A fixed nearest interatomic distance is considered and only nearest neighbor interactions are applied, leading to three force constants $\alpha, \beta$, and $\gamma$ between the atoms $A-A, B-B$, and $A-B$, respectively. For the $2 \mathrm{D}(3 \mathrm{D})$ case, we consider a periodic system characterized with a fixed nearest interatomic distance and a square (cubic) unit cell of dimensions $L \times L(L \times L \times$ $L)$. Inside a unit cell, atoms of type A are arranged in a square area (cubic volume) of size $l \times l(l \times l \times l)$, and atoms of type $\mathrm{B}$ occupy the remaining grid sites. For the $2 \mathrm{D}$ and 3D cases, we consider both nearest and next-nearest neighbor interactions and utilize an inverse square scheme for the next-nearest neighbor force constants. As this is a sandbox approach, we assume $\alpha=\beta=\gamma=1, M_{B}=1$, and discuss results in terms of the mass ratio $m=M_{A} / M_{B}$. We define the extent of the embedded material in terms of the ratio $L_{f}=l / L$, the length fraction along one axis, which is related to packing fraction for 2D (3D) structures by $P_{f}=L_{f}^{2}\left(L_{f}^{3}\right)$.

Our sandbox approach clearly suggests that phononic band gaps are controlled by a combination of five factors: the periodicity $L$, length fraction $L_{f}$, mass ratio $m$, differences in the force constants $\alpha, \beta$, and $\gamma$, and dimensionality of system. The center of a phononic gap decreases as 
$1 / L$ for all dimensionalities with periods larger than 10 atomic monolayers. For smaller periods, there are only minor deviations from this behavior, and, for such PCs, the number of gaps is vastly reduced when compared with PCs with a much larger period, due to a smaller number of atoms in the unit cell. For 1D systems, which only allow one phonon polarization, there is always a phononic gap for $m>1$. In 2D or 3D systems, frequency or polarization gaps are created for the individual transverse (TA) and longitudinal (LA) branches at the zone center and zone edge. These gaps are more clearly identifiable in the low frequency (or acoustic) regime and vary clearly in size as $1 / L$. However, due to the differing velocities of the LA and TA modes, the gaps for these polarizations may not coincide (described as acoustic overlap), and no true phononic gap may result.

In order to increase the frequency gap size, the acoustic mismatch between materials $A$ and $B$ must be increased. This is achievable in three ways: (i) increasing the spring constant ratio $\alpha / \beta$ to an excessively high value, (ii) making the spring constant $\gamma$ either much stronger or weaker than $\alpha$ and $\beta$, and (iii) increasing the mass ratio $m$. Factor (i) is not generally realizable for crystalline structures, and effect (ii) may only be achievable in structures whose constituent materials lead to either a high lattice mismatch or strain effects. From our calculations, we find that regardless of (i) and (ii), the effect of (iii) is highly dependent on the dimensionality of the structure. As noted earlier, a phononic gap is always present for $m>1$ in a 1D system. However, for 2D and 3D systems, the effect of the mass ratio is coupled with the length fraction $L_{f}$, due to acoustic overlap. Our calculations establish the criteria that a true 2D (3D) phononic gap will appear for 2D (3D) systems with $m>10(m>15)$ within the range $0.9>$ $L_{f}>0.1$. A typical example of the phonon dispersion relations is shown in Fig. 1 for $m=25$.

The sandbox approach shows the following trends for PCs: (i) the magnitude of negative group velocity for a given polarization progressively decreases for higher branches, (ii) there is an optimum length fraction dependent on the constituent materials and structure of a system to exhibit phononic properties, (iii) for higher frequencies than the common acoustic range in these structures, confinement and localization effects dominate, and thus these modes have very low group velocities and large frequency gaps, (iv) for 1D or 2D PCs any in-plane gaps may be masked by the folding effects from in-plane vibrational modes.

While the sandbox approach provides valuable insight into the vibrational properties of model PCs, for real semiconductors, it proves insufficient. The failure of such models arises in accurately obtaining bulk phonon eigensolutions, where several parameters (over 20) are required and physical meaning is lost. The adiabatic bond-charge model [9] has successfully been applied to

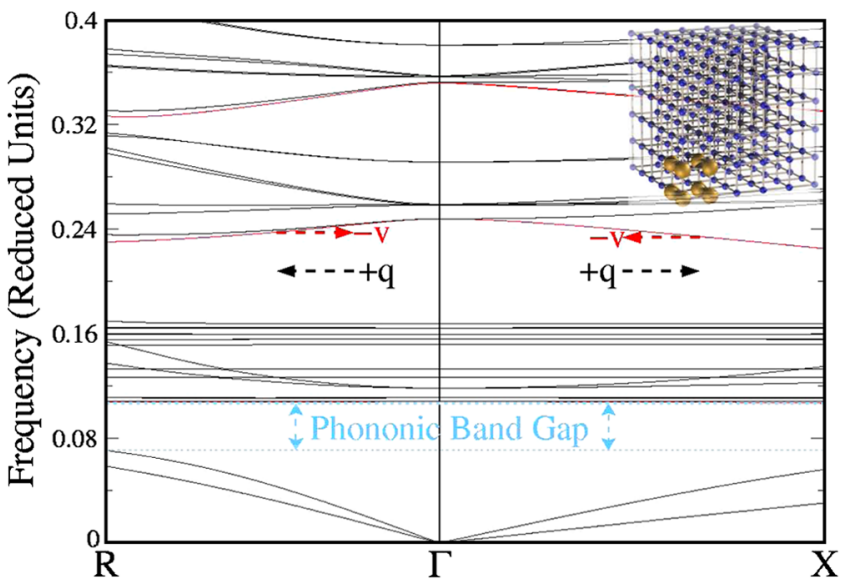

FIG. 1 (color online). Phonon dispersion relations for the sandbox approach containing high mass atoms embedded into a low mass material, with mass ratio of $m=25$. The inset shows the unit cell considered. The low frequency range is shown to highlight the concepts of phononic gap and negative phonon velocity, $-\mathbf{V}$, for positive wave vector $\mathbf{q}$.

study the lattice dynamics of tetrahedrally bonded semiconductors and their nanostructures. In this work, we have applied an enhanced version of the bond-charge model (EBCM) to tetrahedrally bonded $\mathrm{PCs} \mathrm{Si} / \mathrm{Sn}$ and $\mathrm{Si} / \mathrm{Ge}$ by considering the parameters required to calculate the matrix elements for interface bonds as appropriately weighted average values of the bulk materials. For structures containing alloys, such as in $\mathrm{Si}_{x} \mathrm{Ge}_{1-x}$ and $\mathrm{Si} / \mathrm{Si}_{x} \mathrm{Ge}_{1-x}$ superlattices, the parameters of the total system are taken as the weighted average of the corresponding bulk parameters. For $\mathrm{Si}_{x} \mathrm{Ge}_{1-x}$ systems of differing cell sizes with a random distribution of $\mathrm{Si}$ and $\mathrm{Ge}$ atoms, we obtain phonon frequency results that are in very good agreement with the measured results of Brya [10]. Various configurations create a frequency distribution within $\pm 0.06 \mathrm{THz}$ in the phonon dispersion relations. While strain effects may lead to changes in the bond strength, as $\omega \propto \sqrt{\alpha}$, the overall effect is not expected to be significant, especially as modeled parameters provide results which agree with experimental values.

Our EBCM calculations for various semiconducting structures confirm the trends and criteria suggested previously by the sandbox approach. We now apply the EBCM to various composites that have potential as PCs. While the $\mathrm{Si} / \mathrm{Sn}$ system is rather unrealistic to grow in crystalline form due to strain effects, it nevertheless provides useful insight into potential phononic properties. The system consisting of an Sn cubic dot with $L_{f}=0.9$ and $m=$ 4.23 embedded in Si bulk with $L \approx 2.7 \mathrm{~nm}$ exhibits a large frequency gap in the optical range, of $\approx 1 \mathrm{THz}$ centered around $10 \mathrm{THz}$, close to the minimum in the density of states (DOS) of bulk Si. The phonon branches above and below this gap have near zero group velocities, due to the localization of Si modes in the majority Sn environment. 


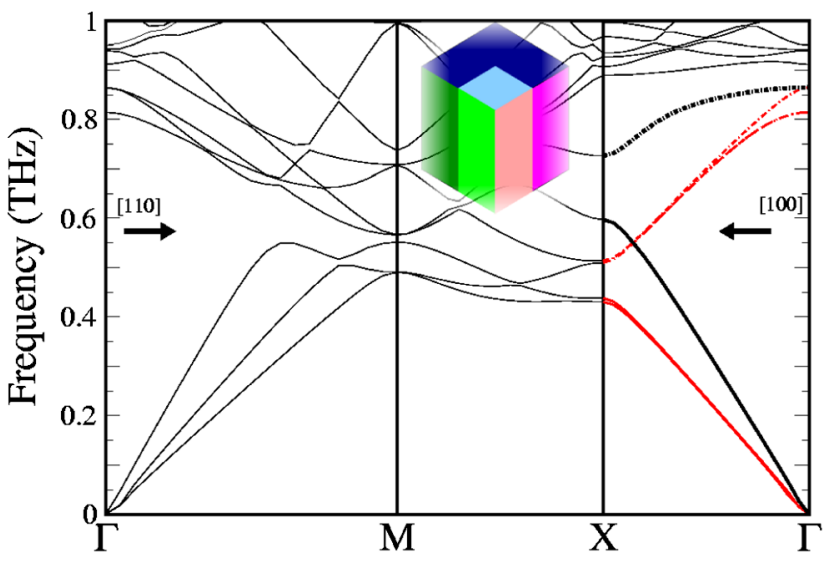

FIG. 2 (color online). Phonon dispersion relations of a cubic Ge pillar $(3.8 \mathrm{~nm})$ embedded in bulk $\mathrm{Si}$ with a period of $5.743 \mathrm{~nm}$. Highlighted are the TA and LA branches and their corresponding folded branches. The inset shows a schematic of the structure.

The second system considered is Sn square towers (grown in the [001] direction) embedded in an Si host with $L_{f}=0.35$ and $L=5.43 \mathrm{~nm}$. At the zone boundary $X$, the lowest folded TA branch (the top end of the TA gap) overlaps the LA branch (the bottom end of the LA gap) by no more than $10 \mathrm{GHz}$. The gap between the lowest folded branch and the highest acoustic branch, along the [110] symmetry direction, remains consistently greater than $60 \mathrm{GHz}$, showing that in this direction, the system is a 1D PC. As a consequence of the disallowed TA modes between 869 and $1050 \mathrm{GHz}$ in all directions, a negative group velocity of the order of $\mathrm{km} / \mathrm{s}$ develops in both the [100] and [110] directions. Also, for values of $L_{f}$ greater or smaller than 0.35 , the acoustic overlap increases. This and further calculations imply that $33 \%$ is the optimum length fraction for a potential semiconducting PC. A comparison of 2D and 3D periodic systems with $m>1$ shows that the length fraction along a particular direction, rather than the packing fraction, is a more meaningful parameter when discussing phononic systems. The directional dependence of the phononic gap obtained from our atomic-scale theory cannot be reproduced from simple continuum theory. Within its applicable limits, the continuum theory [3] relies on the properties of each constituent system being isotropic. While for macroscopic systems this is very suitable, for nanoscale due to the anisotropy of atomic bonds leading to anistropy in phonon dispersion relations (and velocities), this approximation is inadequate. Also, the continuum model is unable to produce the complete phonon frequency spectra.

The corresponding periodic system of Ge pillars embedded in a host of $\mathrm{Si}$ with $L=5.43 \mathrm{~nm}$ and $L_{f}=0.35$ is shown in Fig. 2. This structure shows a minimum in the 2D DOS at $\approx 0.5 \mathrm{THz}$. The folded TA and LA branches (highlighted) have negative group velocities of -5.4 and $-8.4 \mathrm{~km} / \mathrm{s}$, respectively. Similar to the previous $\mathrm{Si} / \mathrm{Sn}$

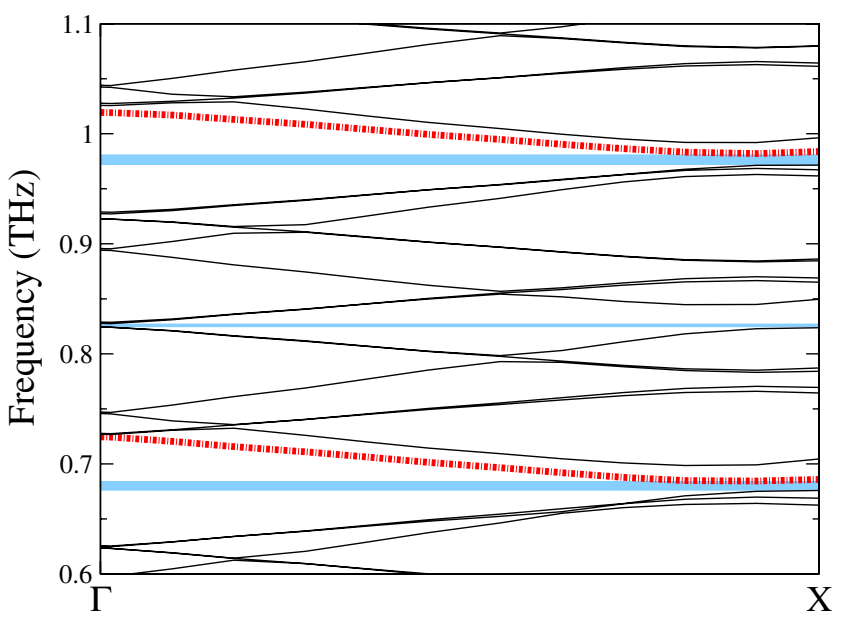

FIG. 3 (color online). Phonon dispersion relations of a $\mathrm{Si}(27 \mathrm{~nm}) / \mathrm{Ge}(17 \mathrm{~nm})$ [100] superlattice. Highlighted are the three gap regions and two folded branches.

structures, this system is a 1D PC with a clear gap from $\Gamma$ to $M$, the least of the high symmetry directions. As discussed earlier using the sandbox approach, strain effects are expected to increase these gaps. For pillars with $L_{f}=$ 0.95 , there is a clear gap between the frequencies 7.79 and $8.39 \mathrm{THz}$, and the optical modes have near zero group velocities. This, as discussed before, is due to localization effects.

Two types of silicon-based superlattice structures have been realized experimentally: $\mathrm{Si} / \mathrm{Ge}$ [11] and $\mathrm{Si} / \mathrm{Si}_{x} \mathrm{Ge}_{1-x}$ [6]. Figure 3 shows our calculated results for a $\mathrm{Si}(27 \mathrm{~nm}) / \mathrm{Ge}(17 \mathrm{~nm})$ [100] superlattice similar to those grown by Lee et al. [11]. It shows three clear gaps in the phonon spectra at 680,826 , and $974 \mathrm{GHz}$, of widths 10,3 ,

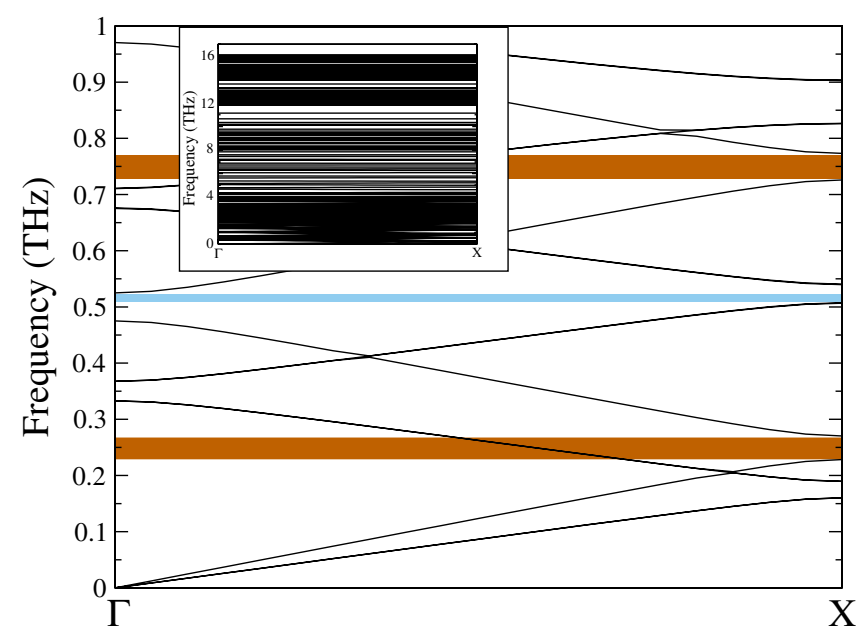

FIG. 4 (color online). Phonon dispersion relations of a $\mathrm{Si}(4 \mathrm{~nm}) / \mathrm{Si}_{0.4} \mathrm{Ge}_{0.6}(8 \mathrm{~nm})$ [100] superlattice. The inset shows the full range for the frequency spectra. Highlighted are the three disallowed LA frequency regions, with the central region showing a true phononic gap. 
TABLE I. The positions of the phonon gaps, their sizes, their polarization characteristics, their zone location $(\mathrm{ZE}=\mathrm{zone}$ edge, $\mathrm{ZC}=$ zone centre $)$ and the velocities of the corresponding adjacent branches for the $\mathrm{Si}(4 \mathrm{~nm}) / \mathrm{Si}_{0.4} \mathrm{Ge}_{0.6}(8 \mathrm{~nm})[100]$ superlattice.

\begin{tabular}{ccccc}
\hline \hline Frequency $(\mathrm{GHz})$ & Gap size $(\mathrm{GHz})$ & Polarization and location & Lower branch velocity $(\mathrm{km} / \mathrm{s})$ & Upper branch velocity $(\mathrm{km} / \mathrm{s})$ \\
\hline 174.8 & 29.5 & TA ZE & 4.18 & -4.18 \\
252.4 & 47.8 & LA ZE & 6.00 & -5.94 \\
350.2 & 34.8 & TA ZC & -4.18 & 4.03 \\
495.1 & 39.7 & LA ZC & -5.94 & 5.84 \\
523.2 & 33.1 & TA ZE & 4.03 & -3.98 \\
805.0 & 30.0 & LA ZE & 5.84 & -5.80 \\
\hline \hline
\end{tabular}

and $10 \mathrm{GHz}$, respectively. The phonon dispersion curves highlighted (dashed curves) in Fig. 3 are doubly degenerate and have maximum negative group velocities of -2.9 and $-2.0 \mathrm{~km} / \mathrm{s}$ for the lower and higher branches, respectively. Also, all phonon modes with frequencies between 688 and $726 \mathrm{GHz}$ have negative group velocities. Comparing this $\mathrm{Si} / \mathrm{Ge}$ structure with $\mathrm{Si} / \mathrm{SiGe}$ systems with similar $L$ and $L_{f}$ reveals that the gaps are larger in the $\mathrm{Si} / \mathrm{Ge}$ due to the larger concentration of perturbing $\mathrm{Ge}$ atoms.

Ezzahri et al. [6] have shown that the $\mathrm{Si}(4 \mathrm{~nm}) /$ $\mathrm{Si}_{0.4} \mathrm{Ge}_{0.6}(8 \mathrm{~nm})$ [100] superlattice exhibits three LA band gaps: $36 \mathrm{GHz}$ gap at $145 \mathrm{GHz}, 46 \mathrm{GHz}$ gap at $283 \mathrm{GHz}$, and $30 \mathrm{GHz}$ gap at $527 \mathrm{GHz}$. However, no information is available for (i) band gaps for TA phonons and (ii) negative group velocities for these branches. Figure 4 shows the complete phonon dispersion relations from our calculations for this superlattice. Several frequency gaps, for both LA and TA polarizations, are obtained in the entire frequency range as shown in the inset. The random disorder of the atomic makeup of the $\mathrm{Si}_{0.4} \mathrm{Ge}_{0.6}$ section leads to an increase in the number of gaps when compared to an ordered system. As expected, confinement effects lead to the optical branches showing very low group velocities. A close examination of Fig. 4 and Table I shows that our results for the LA branch are in good agreement with the experimental results measured by Ezzahri et al. and show, in addition to our presented results for the TA modes, that there is a small frequency range of $8 \mathrm{GHz}$ (between 507 and $515 \mathrm{GHz}$ ) of true phononic gap, in which phonons of neither polarization can propagate. Thus, our calculated results reveal that this system is a true 1D PC.

In conclusion, using a combination of simple and realistic atomic-scale theories, we have examined trends and criteria for phononic properties of composite systems and identified several semiconducting 1D PCs. Our sandbox approach has shown that gap positions and size vary inversely with period, and the size of polarization gaps (and true gaps) increase with a larger mass ratio. These results have been confirmed using the EBCM. Results of the EBCM also show that nanoscale semiconducting compos- ite structures have the potential of being 1D phononics. Embedded towers of $\mathrm{Sn}$ or $\mathrm{Ge}$ in $\mathrm{Si}$ host show a clear hypersonic band gap in the [110] direction. Phonon dispersion relations for nanosized $\mathrm{Si} / \mathrm{Ge}$ and $\mathrm{Si} / \mathrm{SiGe}$ superlattices show several gaps in the hypersonic range in the direction of growth. All these systems are found to have phonons with negative group velocities of the order of $\mathrm{km} / \mathrm{s}$. We have presented the full phonon dispersion relations for the superlattice grown by Ezzahri et al. and have identified it to be a true 1D PC with a clear gap between 507 and $515 \mathrm{GHz}$. Our work clearly suggests that superlattices and other nanoscale semiconductor composite systems present ideal candidates for phonon engineering by tailoring their vibrational properties with controlled system fabrication.

This work has been carried out with financial support from the Leverhulme Trust (UK) through Grant No. F/00 144/AS.

[1] Z. Liu et al., Science 289, 1734 (2000); M. Torres et al., Phys. Rev. Lett. 86, 4282 (2001); M. Siglas and E. N. Economou, J. Sound Vib. 158, 377 (1992); M.S. Kushwaha et al., Phys. Rev. Lett. 71, 2022 (1993); E. L. Thomas et al., Nature Mater. 5, 773 (2006).

[2] S. Yang et al., Phys. Rev. Lett. 93, 024301 (2004).

[3] J. O. Vasseur et al., Phys. Rev. B 77, 085415 (2008); R. Sainidou et al., Phys. Rev. B 66, 212301 (2002).

[4] J. Baumgartl et al., Phys. Rev. Lett. 99, 205503 (2007); T. Still et al., Phys. Rev. Lett. 100, 194301 (2008); T. Gorishnyy et al., Phys. Rev. Lett. 94, 115501 (2005).

[5] J.-F. Robillard et al., Phys. Rev. B 76, 092301 (2007); A. Huynh et al., Phys. Rev. Lett. 97, 115502 (2006).

[6] Y. Ezzahri et al., Phys. Rev. B 75, 195309 (2007).

[7] S. Mohammadi et al., Appl. Phys. Lett. 92, 221905 (2008).

[8] J. S. Jensen, J. Sound Vib. 266, 1053 (2003); P. G. Martinsson and A. B. Movcahn, Q. J. Mech. Appl. Math. 56, 45 (2003).

[9] S. P. Hepplestone and G. P. Srivastava, Nanotechnology 17, 3288 (2006).

[10] W. J. Brya, Solid State Commun. 12, 253 (1973).

[11] S.-M. Lee et al., Appl. Phys. Lett. 70, 2957 (1997). 